\title{
BMJ Open Contrast extravasation and outcome of endovascular therapy in acute ischaemic stroke: a systematic review and meta- analysis
}

Tao Xu, You Wang, Jinxian Yuan, Yangmei Chen, Haiyan Luo

To cite: Xu T, Wang Y, Yuan J, et al. Contrast extravasation and outcome of endovascular therapy in acute ischaemic stroke: a systematic review and meta-analysis. BMJ Open 2021:11:e044917. doi:10.1136/ bmjopen-2020-044917

- Prepublication history and additional online supplemental material for this paper are available online. To view these files, please visit the journal online. To view these files, please visit the journal online (http://dx.doi.org/10.1136/ bmjopen-2020-044917).

Received 17 September 2020 Accepted 24 May 2021
Check for updates

(C) Author(s) (or their employer(s)) 2021. Re-use permitted under CC BY-NC. No commercial re-use. See rights and permissions. Published by BMJ.

Department of Neurology, the Second Affiliated Hospital of Chongqing Medical University, Chongqing, China

Correspondence to

Haiyan Luo;

haiyan|@hospital.cqmu.edu.cn

\section{ABSTRACT}

Objective Contrast extravasation (CE) after endovascular therapy (EVT) is commonly present in acute ischaemic stroke (AIS) patients. Substantial uncertainties remain about the relationship between $\mathrm{CE}$ and the outcomes of EVT in patients with AIS. Therefore, we aimed to evaluate this association.

Design A systematic review and meta-analysis of published studies were performed.

Data source We systematically searched the Medline and Embase databases for relevant clinical studies. The last literature search in databases was performed in June 2020.

Eligibility criteria for study selection We included studies exploring the associations between CE and the outcomes of EVT in patients with AIS undergoing EVT. Data extraction and synthesis Two reviewers extracted relevant information and data from each article independently. We pooled ORs with Cls using a randomeffects meta-analysis to calculate the associations between CE and outcomes of EVT. The magnitude of heterogeneity between estimates was quantified with the $I^{2}$ statistic with $95 \%$ Cls.

Results Fifteen observational studies that enrolled 1897 patients were included. Patients with CE had higher risks of poor functional outcome at discharge $(2.38,95 \%$ Cl 1.45 to $3.89 p=0.001 ; n=545)$ and poor functional outcome at 90 days (OR 2.16, 95\% $\mathrm{Cl} 1.20$ to 3.90; $\mathrm{n}=1194)$. We found no association between CE and inhospital mortality (OR 0.95, 95\% Cl 0.27 to $3.30 ; n=376$ ) or 90-day mortality (OR 1.38, 95\% Cl 0.81 to 2.36 ; $n=697$ ) after EVT. Moreover, CE was associated with higher risks of post-EVT intracranial haemorrhage (ICH) (OR 6.68, 95\% Cl 3.51 to $12.70 ; n=1721$ ) and symptomatic ICH (OR 3.26, $95 \%$ Cl 1.97 to $5.40 ; n=1092$ ).

Conclusions This systematic review and meta-analysis indicates that in patients with AIS undergoing EVT, CE is associated with higher risks of unfavourable functional outcomes and $\mathrm{ICH}$. Thus, we should pay more attention to CE in patients with AIS undergoing EVT.

\section{INTRODUCTION}

Over the past several years, clinical studies have confirmed the efficacy and safety of endovascular therapy (EVT) for treating acute ischaemic stroke (AIS) caused by large
Strengths and limitations of the study

- This study assessed the associations between contrast extravasation (CE) and the clinical outcomes of endovascular therapy (EVT) in patients with acute ischaemic stroke.

- Dual-energy CT (DECT) was considered to be more effective for early differentiation between CE and haemorrhage than nonenhanced computed tomography; however, of the included studies, only four included studies used DECT, which may reduce its diagnostic accuracy for $\mathrm{CE}$, further weakening our results.

- Most of the included studies made a strict distinction between CE and intracranial haemorrhage (ICH); thus, the clinical relevance between the coexistence of $\mathrm{CE}$ and $\mathrm{ICH}$ and the outcomes of EVT remains unclear.

- Most of the included studies included a limited number of subjects, which reduced the strength of this systematic review and meta-analysis.

- The location and volume of CE is an important confounding factor affecting the association between CE and EVT outcomes; however, most of the included studies did not report this key information.

vessel occlusion (LVO). ${ }^{1}$ In recent clinical practice, EVT has been a standard therapy for patients with AIS caused by LVO. ${ }^{1}$ Intravascular injection of iodinated contrast media is commonly administered in EVT. Contrast extravasation (CE) after EVT occurs in some patients with AIS receiving EVT treatment. ${ }^{2}$ CE is usually assessed by a non-enhanced CT (NECT) scan or dual-energy CT (DECT) immediately after EVT and progressively resolves within 24 hours after EVT. ${ }^{34} \mathrm{CE}$ is considered a manifestation of early bloodbrain barrier (BBB) disruption after EVT, which has been reported to be predictive of poor outcome in patients undergoing EVT for AIS. ${ }^{3}$ However, among the studies focusing on the prognosis of patients eligible for EVT with CE, some have indicated that 
patients with CE had a higher risk for impaired functional outcomes, while others found no association between CE and the outcomes of EVT. Thus, this association remains unclear and has substantial uncertainties. Therefore, we aimed to evaluate the association between $\mathrm{CE}$ and the outcomes of EVT in AIS by performing a systematic review and meta-analysis.

\section{METHODS}

\section{Search strategy}

We performed this systematic review and meta-analysis based on the Meta-analysis of Observational Studies in Epidemiology guidelines. ${ }^{5}$ The Medline and Embase databases were systematically searched using a predefined retrieval strategy (online supplemental table 1 in the online data supplement).

\section{Inclusion criteria}

We included a study if it met all of the following inclusion criteria:

1. Exposure and outcome: The study investigated the associations between $\mathrm{CE}$ and the outcomes of EVT for the treatment of AIS.

2. Definition of EVT: EVT was considered endovascular interventional therapy using aspiration techniques, stent retrievers or intra-arterial thrombolysis for the treatment of AIS.

3. Definition of CE: CE was detected with NECT immediately after EVT, and follow-up NECT, MRI T2-weighted gradient-recall echo imaging (MRI-GRE) or MRI susceptibility-weighted-imaging (MRI-SWI) were conducted 24 hours after EVT $^{6}{ }^{7}$; CE was defined as the presence of high density on NECT immediately after EVT but with no discernible high density on 24-hour follow-up NECT after EVT or no hypointensity on 24hour follow-up MRI-GRE and MRI-SWI after EVT. ${ }^{6}$ Moreover, CE could also be detected with DECT. For DECT, CE was defined as exhibiting high density on mixed energy (MIX) images and iodine overlay maps but no high density in the corresponding areas on virtual noncontrast-enhanced (VNC) images. ${ }^{3}$ The differential diagnosis between $\mathrm{CE}$ and cerebral haemorrhage based on neuroimaging is available in table 1 .

4. Outcome definitions: The following outcomes were recorded: poor functional outcome at 90 days (defined as a modified Rankin Scale score (mRS) $\geq 3$ at 90 days after EVT), poor functional outcome at discharge (defined as an $\mathrm{mRS} \geq 3$ at discharge after EVT), in-hospital mortality, 90-day mortality, intracranial haemorrhage (ICH) and symptomatic ICH (sICH) after EVT. PostEVT ICH was detected with CT or MRI scans after CE assessment and was defined as any haemorrhagic event, including haemorrhagic infarction, parenchymal haemorrhage or ICH-extracerebral haemorrhage ${ }^{8}$; sICH was defined as ICH with significant neurological aggravation and an increase in National Institutes of Health Stroke Scale score $\geq 4$ in total. 
5. Assessment of outcome: The study provided the adjusted or unadjusted OR and the corresponding 95\% CI for the magnitude of the association between $\mathrm{CE}$ and each outcome of EVT or provided raw data that could be used to calculate the OR and $95 \%$ CI.

Exclusion criteria: Non-original articles, articles with irrelevant outcomes or insufficient data, or case reports were excluded. A study that did not investigate the associations between $\mathrm{CE}$ and the outcomes of EVT was considered to have irrelevant outcomes. One study without any data regarding outcome assessment after EVT was considered to have insufficient data (did not meet the fifth inclusion criterion). Two authors (TX and YW) performed the literature search independently. Moreover, the reference lists of the included articles were also examined to obtain relevant studies. A disagreement about the inclusion of a study was resolved by us via our discussion until a consensus was reached. We performed last literature search in June 2020. In this meta-analysis, if there was a significant sample overlap among multiple studies, we included the study with the largest sample size or longest follow-up time.

\section{Data extraction and qualitative assessment}

Two reviewers (TX and JY) independently extracted the following data from each article: first author, publication year, territory, study period and design, methods of EVT, demographics of population, sites of vascular lesions, strategies of EVT, and outcomes of EVT. The ORs with $95 \%$ CIs or raw data were extracted to calculate pooled ORs. When a study reported both unadjusted and adjusted ORs, the OR from the most fully adjusted model was extracted. When the effect estimates were not provided directly, the ORs and 95\% CIs were calculated based on raw data (extracted raw data are listed in online supplemental table 2 in the online data supplement). We assessed the quality of the included studies according to the Newcastle-Ottawa scale (NOS).$^{9}$ The full NOS score was 9 stars; if a study awarded $\geq 8$ stars, it was defined as a high-quality study. ${ }^{9}$

\section{Outcome definitions}

The primary outcome of this meta-analysis was poor functional outcome at 90 days after EVT. The secondary outcomes included poor functional outcome at discharge, 90-day mortality, in-hospital mortality, ICH and sICH after EVT.

\section{Statistical analysis}

We used pooled OR to evaluate the magnitude of the association between CE and each outcome of EVT. The magnitude of heterogeneity between estimates was quantified with the $\mathrm{I}^{2}$ heterogeneity test statistic. We also estimated 95\% CIs to assess the magnitude of heterogeneity between estimates. ${ }^{10}$ We recognised the potential heterogeneity and varied underlying effect sizes between the included studies; thus, we used a random effects model to pool the estimates. To examine the sources of heterogeneity, we

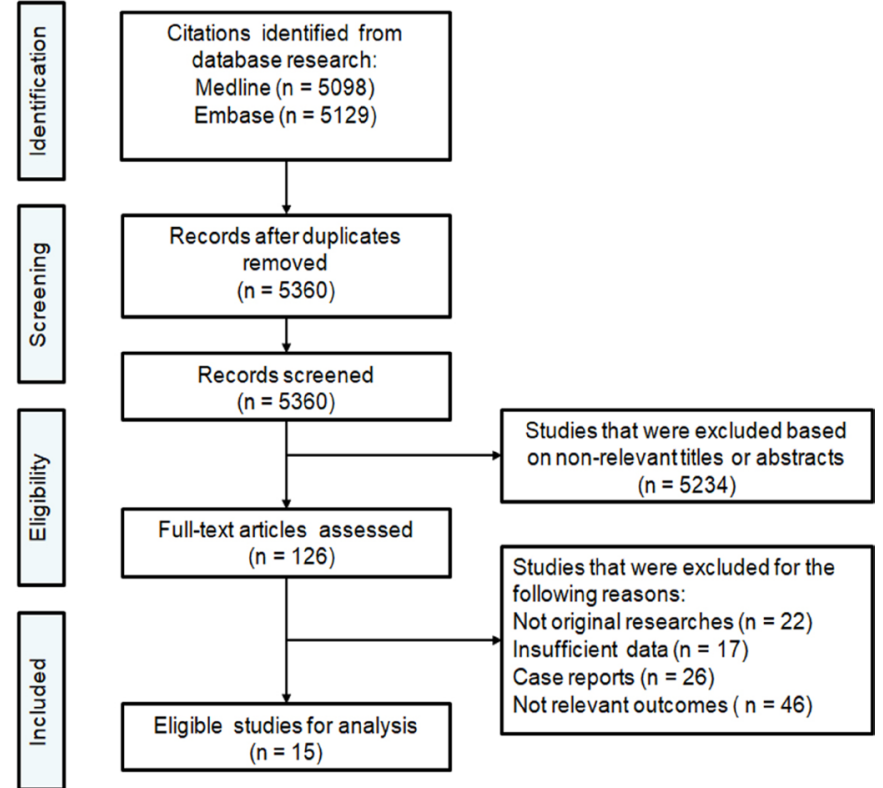

Figure 1 Flow chart of the literature search process.

also performed subgroup analyses based on predefined variables (eg, study design, sample size, $\mathrm{CE}$ assessment strategy, study quality and adjustments for confounders). We also performed meta-regression analyses to assess the influence of predefined variables on the heterogeneity among studies; $\mathrm{P}_{\text {interaction }}$ from meta-regression analyses was used to assess the sources of heterogeneity. We investigated publication bias visually with funnel plots and statistically with Egger's tests ${ }^{11}$ when a pooled estimate included $\geq 5$ studies. STATA V.12.0 (StataCorp) was used for the statistical analyses.

\section{Patient and public involvement}

Patients and/or the public were not involved in the design, conduct, reporting, or dissemination plans of this study.

\section{RESULTS \\ Characteristics and quality assessment of the included studies}

The initial literature search provided 5360 unduplicated records. A total of 15 articles published between 2004 and 2020 including 1897 patients met our inclusion criteria and were finally included in this meta-analysis ${ }^{2-4} 67$ 12-21 (figure 1). Table 1 demonstrates the CE assessment strategies of the included studies. Of the 15 included studies, 11 conducted NECT immediately after EVT and further conducted follow-up NECT, MRI-GRE or MRI-SWI at 24 hours after EVT to assess whether CE had occurred after EVT EV $^{4} 121315$ 17-21; only four studies used DECT immediately after EVT to assess CE after EVT. ${ }^{231416}$ The characteristics of each included study are summarised in table 2. Of the 15 included studies, five were from China ${ }^{2312-14}$, five were from South Korea, ${ }^{4619-21}$ two were from France, ${ }^{17} 18$ one was from the USA, ${ }^{15}$ one was from 


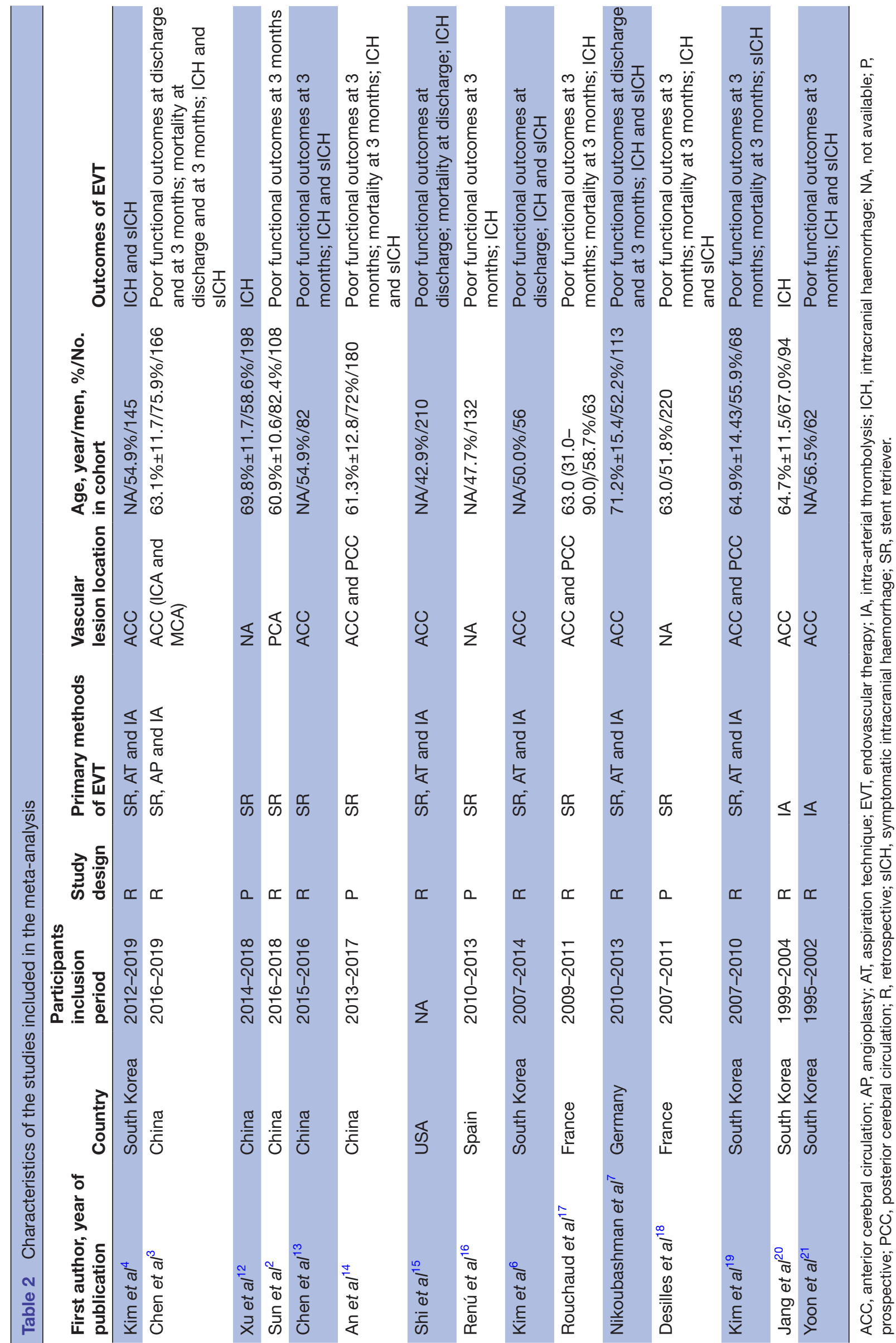




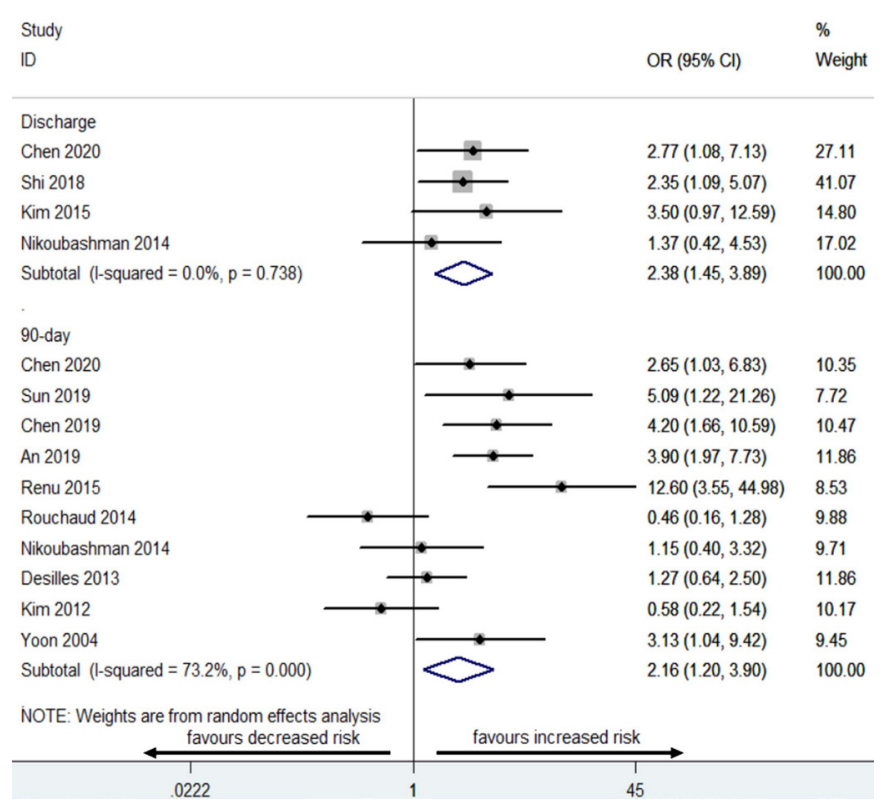

Figure 2 Summary of ORs for the relationships between contrast extravasation (CE) and poor functional outcomes at discharge and 90 days. Each diamond indicates the OR, and the horizontal line indicates the $95 \% \mathrm{Cl}$. CE was associated with higher risks of poor functional outcome at discharge (heterogeneity test: $\mathrm{I}^{2}=0.0 \%, 95 \% \mathrm{Cl} 0.00$ to 0.83 ) and poor functional outcome at 90 days (heterogeneity test: $\mathrm{I}^{2}=73.2 \%$, $95 \% \mathrm{Cl} 0.50$ to 0.86$)$.

Spain $^{16}$ and one was from Germany. ${ }^{7}$ Eleven studies had a retrospective design, ${ }^{2-4} 6713151719-21$ and four had a prospective design. ${ }^{12141618}$ All studies reported that EVT was used for treating AIS, including stent retrievers, aspiration techniques and intra-arterial thrombolysis. Twelve studies reported information on vascular lesion sites: eight studies included patients with AIS caused by LVO in the anterior cerebral circulation (ACC); three studies included patients with AIS caused by LVO in the ACC or posterior cerebral circulation (PCC); and only one study included patients with AIS caused by LVO in the PCC. Most of the included studies had relatively small sample sizes, which ranged from 56 to 220 subjects. The quality assessment of the included studies is summarised in online supplemental table 3 in the online data supplement, and the median score of the included studies was 7.00 (range: 6-9).

\section{The relationship between CE and the outcomes of EVT after AIS}

Regarding the functional outcome after EVT, CE was found to be associated with higher risks of poor functional outcome at discharge (OR 2.38, 95\% CI 1.45 to $3.89 ; \mathrm{p}=0.001 ; 4$ studies; $\mathrm{n}=545)$ and poor 90 -day functional outcome (OR 2.16, 95\% CI 1.20 to 3.90; $\mathrm{p}=0.010 ; 10$ studies; n=1194) (figure 2). However, CE was not related to in-hospital mortality (OR $0.95,95 \%$ CI 0.27 to 3.30 ; $\mathrm{p}=0.934 ; 2$ studies; $\mathrm{n}=376$ ) or 90 -day mortality (OR 1.38, 95\% CI 0.81 to 2.36; $\mathrm{p}=0.232 ; 5$ studies; $\mathrm{n}=697$ ) (figure 3 ). Furthermore, $\mathrm{CE}$ was found to be associated with higher risks for post-EVT ICH (OR 6.68, 95\% CI 3.51 to 12.70; $\mathrm{p}<0.001 ; 13$ studies; $\mathrm{n}=1721$ ) and sICH (OR 3.26, 95\% CI 1.97 to $5.40 ; \mathrm{p}<0.001 ; 9$ studies; $\mathrm{n}=1092$ ) (figure 4 ).

\section{Heterogeneity assessment}

Heterogeneity assessments of pooled estimates were conducted, and the $\mathrm{I}^{2}$ and $95 \%$ CIs are listed in the figure legends. Significant heterogeneity was found in the pooled estimates of poor functional outcome at 90 days $\left(\mathrm{I}^{2}=73.2 \%, 95 \%\right.$ CI 0.50 to 0.86$)$ and post-EVT ICH $\left(\mathrm{I}^{2}=78.80 \%, 95 \%\right.$ CI 0.64 to 0.87$)$. Omitting each study in turn did not alter the significance of pooled estimates and their heterogeneity estimates. Subgroup analyses were performed to assess the relationship between $\mathrm{CE}$ and poor 90-day functional outcome (online supplemental table 4 in the online data supplement) and the association between $\mathrm{CE}$ and post-EVT ICH (online supplemental table 5 in the online data supplement). The results with significant heterogeneity remained stable in subgroup analyses that were restricted to predefined variables. Based on meta-regression analyses, we found that varied assessment strategies of $\mathrm{CE}$ among the included studies accounted for the main between-study heterogeneity $\left(\mathrm{P}_{\text {interaction }}=0.039\right)$ (online supplemental table 4 in the online data supplement).

\section{Publication bias assessment}

Asymmetric funnel plots were identified in the pooled estimates (included $\geq 5$ studies) (online supplemental figures 1-4 in the online data supplement). However, Egger's tests indicated no significant publication bias in

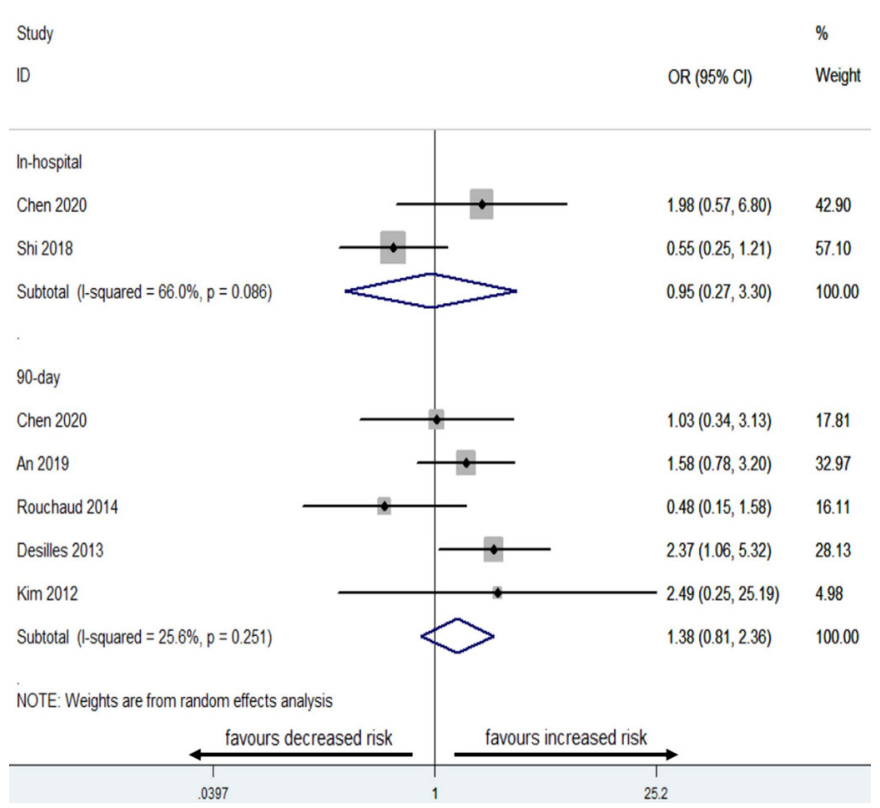

Figure 3 Summary of ORs for the relationships between contrast extravasation (CE) and in-hospital mortality and 90-day mortality. Each diamond indicates the OR, and the horizontal line indicates the $95 \% \mathrm{Cl}$. CE was not associated with in-hospital mortality (heterogeneity test: $I^{2}=66.0 \%, 95 \%$ $\mathrm{Cl}-0.50$ to 0.92 ) or 90 -day mortality (heterogeneity test: $\mathrm{I}^{2}=25.6 \%, 95 \% \mathrm{Cl}-0.85$ to 0.70$)$. 


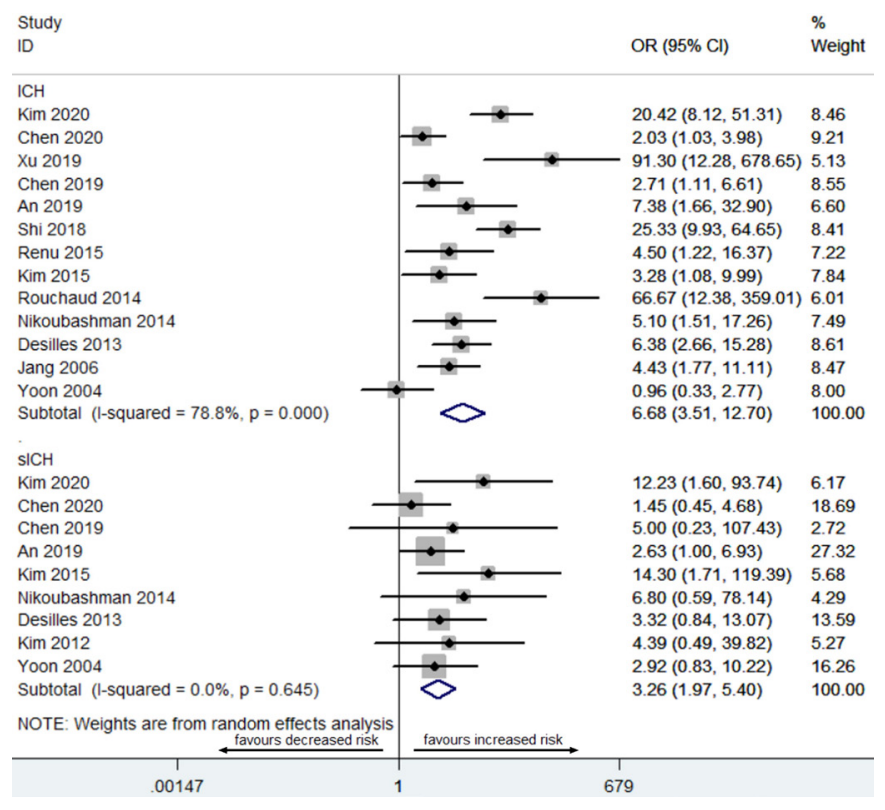

Figure 4 Summary of ORs for the relationships between contrast extravasation (CE) and risks for intracranial haemorrhage $(\mathrm{ICH})$ and symptomatic $\mathrm{ICH}(\mathrm{sICH})$. Each diamond indicates the OR, and the horizontal line indicates the $95 \% \mathrm{Cl}$. CE was related to higher risks of post-EVT ICH (heterogeneity test: $I^{2}=78.8 \%, 95 \% \mathrm{Cl} 0.64$ to 0.87 ) and $\mathrm{sICH}$ (heterogeneity test: $\mathrm{I}^{2}=0.0 \%, 95 \% \mathrm{Cl}-4.30$ to 0.67 ). EVT, endovascular therapy.

the pooled estimates (online supplemental table 6 in the online data supplement).

\section{DISCUSSION}

\section{Main findings}

We performed a systematic review and meta-analysis of the results provided by the 15 included studies having 1897 subjects with EVT for treating AIS caused by LVO. $^{2-4} 67$ 12-21 Our findings based on this meta-analysis indicated that the presence of CE immediately after EVT was related to a higher risk of an unfavourable 90-day functional outcome, indicating that patients with $\mathrm{CE}$ after EVT may have a higher risk of poor functional recovery. Moreover, we found that patients with $\mathrm{CE}$ had higher risks of experiencing ICH and sICH after EVT.

\section{Implications and strength}

The mechanism underlying the clinical relevance of the relationship between $\mathrm{CE}$ and the outcomes of EVT remains unclear. The pathophysiology of CE after EVT is considered to involve a disruption of the $\mathrm{BBB}$ due to initial ischaemia and reperfusion injury. ${ }^{3}{ }^{16}$ In patients with AIS, ischaemic insults can injure vascular endothelial cell junctions and cause damage to the endothelial extracellular matrix, which may promote permeability of the $\mathrm{BBB}$, further allowing for leakage of contrast media into the extravascular space. ${ }^{3}$ Thus, the degree of CE has been reported to be associated with the severity of BBB disruption. In patients undergoing EVT, a delayed reperfusion time (indicating a prolonged ischaemic time) and hyperperfusion after revascularisation may cause greater injury to the vasculature and $\mathrm{BBB}$, further leading to obvious $\mathrm{CE}$ after EVT. ${ }^{16}$ Moreover, procedure-related vascular lesions due to the frequent use of EVT devices and inappropriate operations during EVT may promote BBB disruption. ${ }^{22}$ Additionally, extravasated contrast media may exert direct toxic effects on local brain tissue, which might damage the tissue. ${ }^{316}$ Thus, CE is considered to be associated with poor outcomes after EVT and may have prognostic value in predicting the outcomes of EVT. Thus, therapeutic strategies (such as shortening the recanalisation time, gentle delivery of the EVT device, and controlling blood pressure after EVT) that are able to protect and stabilise the $\mathrm{BBB}$ in the perioperative period of EVT may improve the clinical outcomes of patients with EVT-related CE.

\section{Limitations}

This meta-analysis has several limitations. First, DECT is considered to be more accurate for early differentiation between CE and haemorrhage than NECT. However, of the included studies, only four used DECT, 231416 which may reduce its diagnostic accuracy for CE, further weakening our results. Second, we also noticed the coexistence of CE and haemorrhage immediately after EVT in patients undergoing EVT in clinical practice. However, most of the included studies made a strict distinction between $\mathrm{CE}$ and haemorrhage. Thus, the clinical relevance of the relationship between the coexistence of $\mathrm{CE}$ and haemorrhage and the outcomes of EVT remains unclear. Third, most of the included studies had small sample sizes. Fourth, the location and volume of CE are key confounders influencing the relationship between $\mathrm{CE}$ and the outcomes of EVT in patients with AIS. Most of the included studies, however, did not provide this key information; only two included studies reported that subarachnoid and cortical CE were associated with an elevated risk of ICH. ${ }^{4}$ The effects of $\mathrm{CE}$ location and volume on the relationship between $\mathrm{CE}$ and the outcomes of EVT remain unclear.

\section{CONCLUSIONS}

In summary, in patients undergoing EVT for treating AIS due to LVO, CE was related to elevated risks for unfavourable functional outcomes and ICH events after EVT. Our findings highlight that we should pay careful and increased attention to $\mathrm{CE}$ in patients undergoing EVT for treating AIS due to LVO. Future studies exploring the association between $\mathrm{CE}$ and the outcomes of EVT should take the location and volume of CE into account, which may influence the outcomes of EVT. In this metaanalysis, most eligible studies had retrospective designs; thus, future high-quality prospective studies are needed to explore the association between $\mathrm{CE}$ and the outcomes of EVT.

Contributors HL and YC performed study design; TX and YW performed literature search and selection; TX and JY data acquisition, analysis and interpretation; TX and YW performed statistical analysis; TX and HL drafting of the manuscript. 
Funding This study was supported by the National Science Foundation of China (No. 81901315 and No. 81771390) and also supported by the Kuanren Talents Program of the Second Affiliated Hospital of Chongqing Medical University (No.krycyq-2123), China.

Competing interests None declared.

Patient consent for publication Not required.

Provenance and peer review Not commissioned; externally peer reviewed.

Data availability statement Data are available on reasonable request. The data that support the findings of this study are available from the corresponding author.

Supplemental material This content has been supplied by the author(s). It has not been vetted by BMJ Publishing Group Limited (BMJ) and may not have been peer-reviewed. Any opinions or recommendations discussed are solely those of the author(s) and are not endorsed by BMJ. BMJ disclaims all liability and responsibility arising from any reliance placed on the content. Where the content includes any translated material, BMJ does not warrant the accuracy and reliability of the translations (including but not limited to local regulations, clinical guidelines, terminology, drug names and drug dosages), and is not responsible for any error and/or omissions arising from translation and adaptation or otherwise.

Open access This is an open access article distributed in accordance with the Creative Commons Attribution Non Commercial (CC BY-NC 4.0) license, which permits others to distribute, remix, adapt, build upon this work non-commercially, and license their derivative works on different terms, provided the original work is properly cited, appropriate credit is given, any changes made indicated, and the use is non-commercial. See: http://creativecommons.org/licenses/by-nc/4.0/.

\section{ORCID iD}

Haiyan Luo http://orcid.org/0000-0001-7120-5175

\section{REFERENCES}

1 Román LS, Menon BK, Blasco J, et al. Imaging features and safety and efficacy of endovascular stroke treatment: a meta-analysis of individual patient-level data. Lancet Neurol 2018;17:895-904.

2 Sun Y, Su Y, Chen Z, et al. Contrast extravasation after endovascular treatment in posterior circulation stroke. World Neurosurg 2019;130:e583-7.

3 Chen Z, Zhang Y, Su Y, et al. Contrast extravasation is predictive of poor clinical outcomes in patients undergoing endovascular therapy for acute ischemic stroke in the anterior circulation. J Stroke Cerebrovasc Dis 2020;29:104494.

$4 \mathrm{Kim} \mathrm{H}$, Lee S-J, Lee T-K, et al. Subarachnoid contrast accumulation and Alberta stroke program early computed tomography score applied to contrast accumulation after thrombectomy as predictors of symptomatic hemorrhage. World Neurosurg 2020;138:e847-58.

5 Stroup DF, Berlin JA, Morton SC, et al. Meta-Analysis of observational studies in epidemiology: a proposal for reporting. meta-analysis of observational studies in epidemiology (moose) group. JAMA 2000;283:2008-12.

6 Kim JM, Park KY, Lee WJ, et al. The cortical contrast accumulation from brain computed tomography after endovascular treatment predicts symptomatic hemorrhage. Eur J Neurol 2015;22:1453-8.

7 Nikoubashman O, Reich A, Pjontek R, et al. Postinterventional subarachnoid haemorrhage after endovascular stroke treatment with stent retrievers. Neuroradiology 2014;56:1087-96.

8 von Kummer R, Broderick JP, Campbell BCV, et al. The Heidelberg bleeding classification: classification of bleeding events after ischemic stroke and reperfusion therapy. Stroke 2015;46:2981-6.

9 Stang A. Critical evaluation of the Newcastle-Ottawa scale for the assessment of the quality of nonrandomized studies in metaanalyses. Eur J Epidemiol 2010;25:603-5.

10 von Hippel PT. The heterogeneity statistic I(2) can be biased in small meta-analyses. BMC Med Res Methodol 2015;15:35.

11 Egger M, Davey Smith G, Schneider M, et al. Bias in meta-analysis detected by a simple, graphical test. BMJ 1997;315:629-34.

12 Xu C, Zhou Y, Zhang R, et al. Metallic Hyperdensity sign on noncontrast CT immediately after mechanical thrombectomy predicts parenchymal hemorrhage in patients with acute large-artery occlusion. AJNR Am J Neuroradiol 2019;40:661-7.

13 Chen $\mathrm{W}-\mathrm{H}, \mathrm{Yi}$ T-Y, Wu Y-M, et al. Parenchymal hyperdensity on C-arm CT images after endovascular therapy for acute ischaemic stroke predicts a poor prognosis. Clin Radiol 2019;74:399-404.

$14 \mathrm{An} \mathrm{H}$, Zhao W, Wang J, et al. Contrast staining may be associated with intracerebral hemorrhage but not functional outcome in acute ischemic stroke patients treated with endovascular thrombectomy. Aging Dis 2019;10:784-92.

15 Shi Z-S, Duckwiler GR, Jahan R, et al. Early blood-brain barrier disruption after mechanical thrombectomy in acute ischemic stroke. $J$ Neuroimaging 2018;28:283-8.

16 Renú A, Amaro S, Laredo C, et al. Relevance of blood-brain barrier disruption after endovascular treatment of ischemic stroke: dualenergy computed tomographic study. Stroke 2015;46:673-9.

17 Rouchaud A, Pistocchi S, Blanc R, et al. Predictive value of flat-panel CT for haemorrhagic transformations in patients with acute stroke treated with thrombectomy. J Neurointerv Surg 2014;6:139-43.

18 Desilles J-P, Rouchaud A, Labreuche J, et al. Blood-Brain barrier disruption is associated with increased mortality after endovascular therapy. Neurology 2013;80:844-51.

19 Kim J-T, Heo S-H, Cho B-H, et al. Hyperdensity on non-contrast CT immediately after intra-arterial revascularization. $J$ Neurol 2012;259:936-43.

20 Jang YM, Lee DH, Kim HS, et al. The fate of high-density lesions on the non-contrast CT obtained immediately after intra-arterial thrombolysis in ischemic stroke patients. Korean J Radiol 2006;7:221-8.

21 Yoon W, Seo JJ, Kim JK, et al. Contrast enhancement and contrast extravasation on computed tomography after intraarterial thrombolysis in patients with acute ischemic stroke. Stroke 2004;35:876-81.

22 Shi Z-S, Liebeskind DS, Loh Y, et al. Predictors of subarachnoid hemorrhage in acute ischemic stroke with endovascular therapy. Stroke 2010;41:2775-81. 